Fernando Mazzone*, Departamento de Matemática, Universidad Nacional de Río Cuarto, 5800 - Río Cuarto, Argentina, e-mail: fmazzone@@unrccc.edu.ar

\title{
A CHARACTERIZATION OF ALMOST EVERYWHERE CONTINUOUS FUNCTIONS
}

\begin{abstract}
Let $(X, d)$ be a separable metric space and $\mathcal{M}(X)$ the set of probability measures on the $\sigma$-algebra of Borel sets in $X$. In this paper we will show that a function $f$ is almost everywhere continuous with respect to $\mu \in \mathcal{M}(X)$ if and only if $\lim _{n \rightarrow \infty} \int_{X} f d \mu_{n}=\int_{X} f d \mu$, for all sequences $\left\{\mu_{n}\right\}$ in $\mathcal{M}(X)$ such that $\mu_{n}$ converges weakly to $\mu$.
\end{abstract}

\section{Introduction and Main Result}

Let $(X, d)$ be a metric space. By $\mathcal{M}(X)$ we denote the set of probability measures on $\mathcal{B}_{X}$, where $\mathcal{B}_{X}$ is the $\sigma$-algebra generated by the closed subsets of $X$. Let $\mu \in \mathcal{M}(X)$. We say that a measurable function $f: X \rightarrow \mathbb{R}$ is continuous almost everywhere $(\mu)$ (continuous a.e. $(\mu)$ ) if for the set $D_{f}$ of discontinuity points of $f$ we have $\mu\left(D_{f}\right)=0$, where $D_{f} \in \mathcal{B}_{X}$. If $\left\{\mu_{n}\right\}_{n \in \mathbb{N}}$ is a sequence in $\mathcal{M}(X)$ and $\mu \in \mathcal{M}(X)$, we say that $\left\{\mu_{n}\right\}_{n \in \mathbb{N}}$ converges weakly to $\mu\left(\mu_{n} \rightarrow \mu\right)$ if for any continuous bounded function $f$ on $X$ we have

$$
\lim _{n \rightarrow \infty} \int_{X} f d \mu_{n}=\int_{X} f d \mu
$$

In this paper we will show that a bounded measurable function $f$ is continuous a.e. $(\mu)$ if and only if (1) is fulfilled for any sequence $\left\{\mu_{n}\right\}$ such that $\mu_{n} \rightarrow \mu$.

If $\mu_{n} \rightarrow \mu$, then for each bounded lower semicontinuous (upper semicontinuous) function $h$ we have

$$
\liminf _{n \rightarrow \infty} \int_{X} h d \mu_{n} \geq \int_{X} h d \mu \quad\left(\limsup _{n \rightarrow \infty} \int_{X} h d \mu_{n} \leq \int_{X} h d \mu\right)
$$

Key Words: weak convergence, almost everywhere continuous functions, metric space

Mathematical Reviews subject classification: Primary: 28A60

Received by the editors October 17, 1994

*The author was supported by CONICOR and Universidad Nacional de Río Cuarto. 
see $[1$, p. 17].

A set $B \in \mathcal{B}_{X}$ is called a continuity set for $\mu \in \mathcal{M}(X)$ if $\mu(\partial B)=0$, where $\partial B$ is the boundary of $B$. Let $C_{\mu}$ be the class of all continuity sets. Then $C_{\mu}$ is an algebra, see [3, p. 50]. For any function $g$ on $X$ we define the following functions:

$$
\begin{aligned}
& \bar{g}(x):=\limsup _{r \rightarrow 0}\{g(y): d(y, x)<r\} \\
& \underline{g}(x):=\liminf _{r \rightarrow 0}\{g(y): d(y, x)<r\} .
\end{aligned}
$$

It is well known that $\underline{g}$ and $\bar{g}$ satisfy the following properties:

(i) $\underline{g} \leq g \leq \bar{g}$. Furthermore if $g$ is bounded, then $\underline{g}$ and $\bar{g}$ are bounded.

(ii) $g(\bar{g})$ is a lower semicontinuous (upper semicontinuous) function. Therefore $\underline{g}$ and $\bar{g}$ are measurable functions.

(iii) $\underline{g}(x)=\bar{g}(x)$ if and only if $x$ is a continuity point of $g$.

From (i) and (iii) it follows that $D_{g}=\{x: \bar{g}(x)-\underline{g}(x)>0\}$. Then by (ii) $D_{g} \in \mathcal{B}_{X}$ for any measurable function $g$.

Theorem 1 Let $(X, d)$ be a separable metric space and $\mu \in \mathcal{M}(X)$. We suppose that $f$ is a bounded measurable function on $X$. The following are equivalent:

(a) $f$ is continuous a.e. $(\mu)$.

(b) $\lim _{n \rightarrow \infty} \int_{X} f d \mu_{n}=\int_{X} f d \mu$, for any sequence $\left\{\mu_{n}\right\}_{n \in \mathbb{N}}$ in $\mathcal{M}(X)$ such that $\mu_{n} \rightarrow \mu$.

Proof. We suppose that $f$ is continuous a.e. $(\mu)$ and $\mu_{n} \rightarrow \mu$. Then by (i)-(iii) we obtain

$$
\limsup _{n \rightarrow \infty} \int_{X} f d \mu_{n} \leq \limsup _{n \rightarrow \infty} \int_{X} \bar{f} d \mu_{n} \leq \int_{X} \bar{f} d \mu=\int_{X} f d \mu
$$

and

$$
\liminf _{n \rightarrow \infty} \int_{X} f d \mu_{n} \geq \liminf _{n \rightarrow \infty} \int_{X} \underline{f} d \mu_{n} \geq \int_{X} \underline{f} d \mu=\int_{X} f d \mu .
$$

Hence we get (b).

Now we suppose that (b) holds. We will show that there is a sequence $\left\{\mu_{n}\right\}$ in $\mathcal{M}(X)$ such that $\mu_{n} \rightarrow \mu$ and $\lim _{n \rightarrow \infty} \int_{X} f d \mu_{n}=\int_{X} \bar{f} d \mu$. Hence, by (i), $\bar{f}=f$ a.e. $(\mu)$. Since a similar fact holds with $\underline{f}$, we will get that $\bar{f}=\underline{f}$ a.e. $(\mu)$; i.e., $f$ is continuous a.e. $(\mu)$. 
For each $x \in X$ and $r \in \mathbb{R}, B(X, r)$ denotes the open ball of radius $r$ and center $x$. Let $n \in \mathbb{N}$ and $x \in X$. Since the set $\{r: \mu(\partial B(x, r)>0)\}$ is at most countable, there exists a positive real number $r_{x}^{n}$ such that $r_{x}^{n} \leq \frac{1}{n}$ and $B\left(x, r_{x}^{n}\right) \in C_{\mu}$. As $X$ is a separable metric space and $X=\cup_{x \in X} B\left(x, r_{x}^{n}\right)$, there exists a sequence $\left\{x_{j}^{n}\right\}_{j \in \mathbb{N}}$ in $X$ such that $X=\cup_{j=1}^{\infty} B\left(x_{j}^{n}, r_{x_{j}^{n}}^{n}\right)$. We define the sets $A_{j}^{n}$ by $A_{1}^{n}:=B\left(x_{1}^{n}, r_{x_{1}^{n}}^{n}\right)$ and $A_{j}^{n}:=B\left(x_{j}^{n}, r_{x_{j}^{n}}^{n}\right) \backslash\left\{B\left(x_{1}^{n}, r_{x_{1}^{n}}^{n}\right) \cup\right.$ $\left.\cdots \cup B\left(x_{j-1}^{n}, r_{x_{j-1}^{n}}^{n}\right)\right\}$ for $j>1$. The sequence $\left\{A_{j}^{n}\right\}_{j \in \mathbb{N}}$ satisfies

(i) $X=\cup_{j=1}^{\infty} A_{j}^{n}$ and $A_{j}^{n} \in C_{\mu}$. Furthermore $A_{j}^{n} \cap A_{i}^{n}=\emptyset$ if $i \neq j$.

(ii) $\operatorname{diam}\left(A_{j}^{n}\right) \leq \frac{2}{n}$.

For each $A_{j}^{n}$ let $z_{j}^{n} \in A_{j}^{n}$ such that $\sup \left\{f(x): x \in A_{j}^{n}\right\}-\frac{1}{n}<f\left(z_{j}^{n}\right)$. We define the measures $\mu_{n}$ on $\mathcal{B}(X)$ by $\mu_{n}:=\sum_{j=1}^{\infty} \mu\left(A_{j}^{n}\right) \delta_{z_{j}^{n}}$ where as usual $\delta_{x}$ is the Dirac delta measure. Clearly we have that $\lim _{n \rightarrow \infty} \int_{X} g d \mu_{n}=\int_{X} g d \mu$ for each bounded uniformly continuous function $g$. Hence by [3, p. 40] we get that $\mu_{n} \rightarrow \mu$.

Let $A^{0}$ denote the interior of the set $A$. It is easy to prove that if $x \in\left(A_{j}^{n}\right)^{0}$, then $\bar{f}(x)<\bar{f}\left(z_{j}^{n}\right)+\frac{1}{n}$. As $A_{j}^{n} \in C_{\mu}$, we have that

$$
\int_{X} \bar{f} d \mu-\int_{X} f d \mu_{n}=\sum_{j=1}^{\infty} \int_{\left(A_{j}^{n}\right)^{0}}\left\{\bar{f}-\bar{f}\left(z_{j}^{n}\right)\right\} d \mu \leq \frac{1}{n} \sum_{j=1}^{\infty} \mu\left(A_{j}^{n}\right)=\frac{1}{n} .
$$

Therefore

$$
\int_{X} \bar{f} d \mu \geq \int_{X} f d \mu=\lim _{n \rightarrow \infty} \int_{X} f d \mu_{n} \geq \int_{X} \bar{f} d \mu_{n} .
$$

Hence

$$
\int_{X} \bar{f} d \mu=\int_{X} f d \mu
$$

So the proof is complete.

Remark. Obviously the last Theorem is true for $\mu_{n}, \mu$ finite and positive measures.

\section{References}

[1] P. Billingsley, Convergence of Probability Measures, Wiley, New York, 1968.

[2] H. Bergstrom, Weak Convergence of Measures, Academic Press, New York, 1982.

[3] K. R. Parthasarathy, Probability Measures on Metric Spaces, Academic Press, New York, 1967. 